\title{
BMJ
}

\section{Association between radiographic features of knee osteoarthritis and pain: results from two cohort studies}

\author{
Tuhina Neogi, assistant professor of medicine, ${ }^{1}$ David Felson, professor of medicine, ${ }^{1}$ lingbo Niu, research \\ assistant professor of medicine, ${ }^{1}$ Michael Nevitt, professor of medicine, ${ }^{2}$ Cora E Lewis, professor of medicine, ${ }^{3}$ \\ Piran Aliabadi, professor of medicine, ${ }^{4}$ Burt Sack, clinical professor of medicine, ${ }^{1}$ James Torner, professor of \\ medicine, ${ }^{5}$ Lawrence Bradley, professor of medicine, ${ }^{3}$ Yuqing Zhang, professor of medicine ${ }^{1}$
}

${ }^{1}$ Clinical Epidemiology Research and Training Unit, Boston

University School of Medicine, 650 Albany Street, Boston, MA 02118, USA

${ }^{2}$ Department of Epidemiology and Biostatistics, University of

California at San Francisco, San

Francisco, CA 94107-1762, USA

${ }^{3}$ Division of Preventive Medicine, University of Alabama at

Birmingham, Birmingham, AL 35205, USA

${ }^{4}$ Department of Radiology, Brigham and Women's Hospital, Boston, MA 02115, USA

${ }^{5}$ University of lowa, College of Public Health, E107 General Hospital, lowa City, IA 52242, USA Correspondence to: T Neogi tneogi@bu.edu

Cite this as: $B M J$ 2009;339:b2844 doi:10.1136/bmj.b2844

\section{ABSTRACT}

Objective To examine the relation of radiographic features of osteoarthritis to knee pain in people with knees discordant for knee pain in two cohorts.

Design Within person, knee matched, case-control study. Setting and participants Participants in the Multicenter Osteoarthritis (MOST) and Framingham Osteoarthritis studies who had knee radiographs and assessments of knee pain.

Main outcome measures Association of each pain measure (frequency, consistency, and severity) with radiographic osteoarthritis, as assessed by Kellgren and Lawrence grade (0-4) and osteophyte and joint space narrowing grades (0-3) among matched sets of two knees within individual participants whose knees were discordant for pain status.

Results 696 people from MOST and 336 people from Framingham were included. Kellgren and Lawrence grades were strongly associated with frequent knee pain - for example, for Kellgren and Lawrence grade $4 \mathrm{v}$ grade 0 the odds ratio for pain was 151 (95\% confidence interval 43 to 526 ) in MOST and 73 (16 to 331) in Framingham (both $\mathrm{P}<0.001$ for trend). Similar results were also seen for the relation of Kellgren and Lawrence scores to consistency and severity of knee pain. Joint space narrowing was more strongly associated with each pain measure than were osteophytes.

Conclusions Using a method that minimises between person confounding, this study found that radiographic osteoarthritis and individual radiographic features of osteoarthritis were strongly associated with knee pain.

\section{INTRODUCTION}

Pain is the major clinical symptom in osteoarthritis of the knee and a key determinant for seeking medical care. Pain related to osteoarthritis of the knee not only contributes to functional limitations and reduced quality of life but is also the leading cause of impairment of mobility in the elderly population in the United States. ${ }^{1}$ Despite the importance of pain in knee osteoarthritis, little is understood about its causes. The general opinion is that only a modest association exists between radiographic features of osteoarthritis and knee pain, ${ }^{2-4}$ particularly for mild radiographic osteoarthritis. Several investigators have shown discordance between these two features of osteoarthritis: people with clearly abnormal joint radiographs may have no or only mild pain, ${ }^{45}$ whereas others with pain may not have radiographic osteoarthritis, ${ }^{6}$ although this discordance is thought to be less with more severe stages of radiographic disease. ${ }^{78}$ Furthermore, although pain has been associated with osteophytes on plain radiographs, ${ }^{9-12}$ it has generally not been associated with joint space narrowing. ${ }^{911-13}$

Previous studies have shown a lack of high concordance between pain symptoms and radiographic osteoarthritis, but such findings should not be considered as evidence of a lack of causal association. A factor can be strongly causally associated with an outcome, yet it may not be a strong predictor of the outcome on its own because several other factors may contribute to the outcome. This is particularly relevant to the study of pain, which is a subjective experience and unique to each person. Many factors, such as genetic predisposition, ${ }^{1415}$ previous experience, ${ }^{1617}$ expectations about analgesic treatment, ${ }^{18}{ }^{19}$ current mood, ${ }^{20}$ coping strategies (such as catastrophising), ${ }^{21}$ and sociocultural environment, ${ }^{22-24}$ contribute to a person's response to pain. These factors, which usually differ from person to person, are often neither measured nor controlled for in studies examining the relation of pain to radiographic osteoarthritis across individual patients. Consequently, residual confounding may have diluted the association between radiographic knee osteoarthritis and knee pain.

We examined the relation of radiographic osteoarthritis to knee pain among participants from the Multicenter Osteoarthritis (MOST) study and the Framingham Osteoarthritis Study who had knees that were discordant for pain (that is, one knee had pain but the other did not). Specifically, we compared the presence of radiographic features (grade of radiographic osteoarthritis, osteophytes, and joint space narrowing) between the naturally paired knees. This approach eliminated confounders at the level of the participant (such as sociodemographic, genetic, 
psychological, and physiological factors), either known or unknown, and provided more valid estimates of the effect of radiographic features on knee pain.

\section{METHODS}

Study populations

The Multicenter Osteoarthritis (MOST) study is a prospective cohort study of 3026 people aged 50 to 79 years that aims to identify risk factors for incident symptomatic knee osteoarthritis and progressive osteoarthritis in a sample either with or at high risk of osteoarthritis. All MOST participants were recruited from two communities in the United States-Birmingham, Alabama, and Iowa City, Iowa. Details of the study population have been published elsewhere. ${ }^{25}$ In brief, people considered at high risk of developing knee osteoarthritis included those who were overweight or obese and those with knee pain, aching, or stiffness on most of the previous 30 days, a history of knee injury that made it difficult to walk for at least one week, or previous knee surgery.

The Framingham Osteoarthritis Study, a community based study of osteoarthritis, included members of the original cohort from the Framingham Heart Study, the Framingham Offspring Study, and a new cohort recruited by random digit dialling from Framingham, Massachusetts. ${ }^{26}$ Participants of the original Framingham Heart Study, begun in 1948, had knee radiographs taken during the 20th biennial examination (1992-3). Participants in the Framingham Offspring Study included surviving descendants and spouses of descendants of participants in the original Framingham Heart Study. Selection of participants for all Framingham Osteoarthritis study groups was not based on the presence or absence of knee osteoarthritis or knee pain. Details of the study population have been published elsewhere. ${ }^{727}$

Both studies (MOST and Framingham) excluded people with bilateral total knee replacement or rheumatoid arthritis. Rheumatoid arthritis was assessed by using a validated survey instrument, ${ }^{28}$ supplemented by questions about drug use that reflected treated disease. MOST also excluded people who had ankylosing spondylitis, psoriatic arthritis, reactive arthritis, or a history of cancer (except for non-melanoma skin cancer); needed dialysis; were unable to walk without the help of another person or a walker; or planned to move out of the area in the subsequent three years.

\section{Radiographic assessment}

All participants in MOST had bilateral weight bearing, fixed flexion posteroanterior and lateral radiographic evaluation of the knee, as described elsewhere. ${ }^{29}$ In Framingham, all participants in the original, offspring, and community cohorts had similar bilateral weight bearing posteroanterior radiographs, and the offspring and community cohorts also had lateral fixed flexion radiographic evaluation according to a published protocol. ${ }^{30}$ Radiographs in both the MOST and Framingham cohorts were scored by a musculoskeletal radiologist and a rheumatologist blinded to pain status, both experienced in reading study films. Each knee joint was scored for Kellgren and Lawrence grade (04), maximal osteophyte grade (0-3), and maximal joint space narrowing grade (0-3). ${ }^{29}$ Maximal osteophyte and joint space narrowing grades were determined on the posteroanterior and lateral views, thereby including both the tibiofemoral and the patellofemoral joints. The inter-rater reliability (weighted $\kappa$ ) for the Kellgren and Lawrence grade was 0.90 in MOST. For Framingham, readings were adjudicated by three readers when the two primary readers disagreed about the readings.

\section{Assessment of pain}

We used three measures to characterise knee pain. These were presence of frequent knee pain, consistency of frequent knee pain, and severity of knee pain.

\section{Frequent knee pain}

In MOST, all participants were asked a knee specific question about knee pain at the clinic visit: "During the past 30 days, have you had pain, aching, or stiffness in your knee on most days?" In the Framingham Osteoarthritis Study, all participants were asked about knee symptoms at the clinic visit with the following questions: "In the past 30 days, have you had pain, aching or stiffness on most days in either of your knees?" followed by, "Was the pain, aching or stiffness in your right knee, left knee or both knees?" if the response to the first question was positive. For both cohorts, positive responses to the pain questions were considered to indicate the presence of frequent knee pain; negative responses were considered to indicate the absence of frequent knee pain.

\section{Consistency of frequent knee pain}

In MOST, participants were asked the same frequent knee pain question as described above during a telephone screen before the clinic visit, on average 33 days before the visit. A knee was considered to have consistent frequent knee pain if it had frequent knee pain at both the telephone screen and clinic visit or to have inconsistent frequent knee pain if frequent knee pain was present at only one of the telephone screen or clinic visit. This pain measure was not assessed among participants of Framingham.

\section{Severity of knee pain}

Participants of MOST completed a knee specific Western Ontario and McMaster Universities Osteoarthritis Index (WOMAC) pain questionnaire, ${ }^{31}$ a validated instrument to assess knee pain, for both knees at the clinic visit. Severity of knee pain was determined from the WOMAC pain subscale (0-20 scale). A knee specific WOMAC pain questionnaire was not administered in Framingham.

\section{Statistical analyses}

We did separate analyses for MOST and Framingham for analysis of frequent knee pain. The other two pain measures were only assessed in MOST. We identified 
people who had knees that were discordant for each pain measure separately. Two such knees within a person then formed a matched set for a specific pain measurement.

Specifically, for the analysis of the association of radiographic osteoarthritis with presence of frequent knee pain, eligible participants were those who had frequent knee pain in one knee but not the contralateral knee. Likewise, for evaluation of the relation of radiographic knee osteoarthritis to the consistency of frequent knee pain, eligible participants were those who had knees that were discordant in their consistency of frequent knee pain as follows: one knee had consistent frequent knee pain, and the contralateral knee had no frequent knee pain; one knee had inconsistent frequent knee pain, and the contralateral knee had no frequent knee pain; or one knee had consistent frequent knee pain, and the contralateral knee had inconsistent frequent knee pain.

For severity of knee pain, we first classified knees into three categories of pain on the basis of the maximal WOMAC score on any of the five pain questions: severe to extreme pain (maximal WOMAC score of 3 or 4 on any of the five pain questions), mild to moderate pain (maximal WOMAC score of 1 or 2 on any of the five pain questions), and no pain (maximal WOMAC score of 0 on all five pain questions). We then selected participants whose knees were discordant in categories of pain severity: severe to extreme pain in one knee and mild to moderate pain in the contralateral knee; severe to extreme pain in one knee and no pain in the contralateral knee; or mild to moderate pain in one knee and no pain in the contralateral knee.

In separate analyses, we further identified participants in whom both knees had some pain but one knee had greater pain severity than the other knee (that is, discordant for knee pain severity). Specifically, we identified people in whom both knees had non-zero WOMAC pain scores but whose WOMAC scores differed between the knees by at least $20 \%$ with an absolute difference of at least 2 (on a $0-20$ scale).

We examined the relation of each radiographic measure of osteoarthritis (Kellgren and Lawrence grade, maximal osteophyte grade, and maximal joint space narrowing grade) to the prevalence of each of the pain measurements (presence of frequent knee pain, consistency of knee pain, and severity of knee pain) by using conditional logistic regression because of the matched nature of the data. ${ }^{32}$ As two knees within a person formed a matched set, the effects of all person

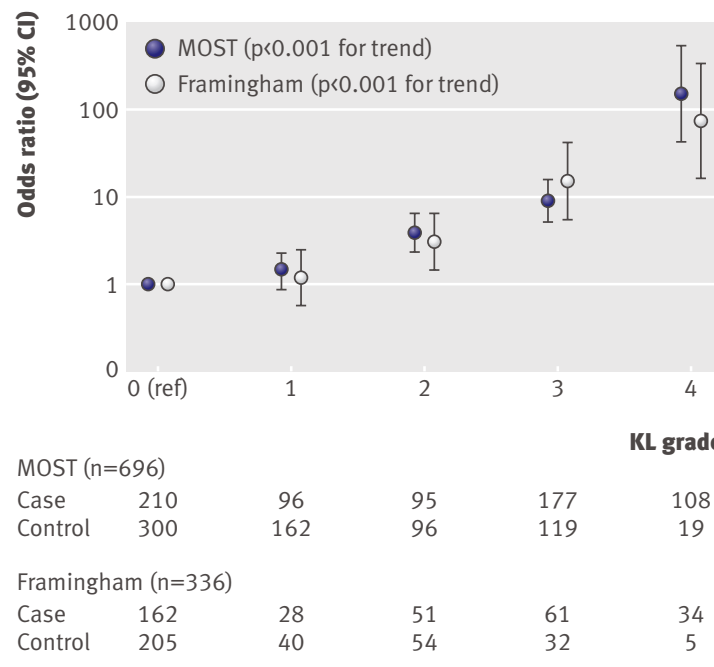

Fig 1| Associations of frequent knee pain with Kellgren and Lawrence (KL) grade among people with two knees discordant for frequent knee pain status. No of case knees (those with frequent knee pain) and control knees (those without frequent knee pain) shown beneath graph for each $\mathrm{KL}$ grade. Note that $\mathrm{y}$ axis is logarithmically scaled

level confounders (such as age, sex, body mass index) were implicitly eliminated. To evaluate the effects of severity of osteophyte and joint space narrowing, we entered both into the regression models to mutually adjust for one another. We used SAS 9.1 for all analyses.

\section{RESULTS}

We identified 696 people from MOST and 336 people from Framingham as having knees discordant for presence of frequent knee pain. For these participants in MOST, $418(60 \%)$ were female, the mean age was 62 (SD 8, range 50-79) years, and the mean body mass index was 31 (SD 6, range 18-56). In Framingham, $208(62 \%)$ of these participants were female, the mean age was 68 (SD 10, range 49-93), and the mean body mass index was 29 (SD 5, range 17-58).

Tables 1 and 2 show the distribution of Kellgren and Lawrence grades for the paired knees within people who were discordant in their frequent knee pain status in the MOST and Framingham studies. Both tables show that knees with frequent pain were more likely to have higher Kellgren and Lawrence grades than were the contralateral knees without frequent pain (upper right hand parts of the tables). In MOST, knees with Kellgren and Lawrence grades 1, 2, 3, and

Table 1| Kellgren and Lawrence (KL) grade frequencies for pairs of knees discordant for frequent knee pain in MOST (n=696)

\begin{tabular}{|c|c|c|c|c|c|}
\hline \multirow[b]{2}{*}{$\mathrm{KL}$ grades for knees without frequent knee pain } & \multicolumn{5}{|c|}{$\mathrm{KL}$ grades for knees with frequent knee pain } \\
\hline & 0 & 1 & 2 & 3 & 4 \\
\hline 0 & 176 & 28 & 36 & 40 & 20 \\
\hline 1 & 22 & 48 & 29 & 45 & 18 \\
\hline 2 & 10 & 10 & 24 & 35 & 17 \\
\hline 3 & 2 & 10 & 16 & 54 & 37 \\
\hline 4 & 0 & 0 & 0 & 3 & 16 \\
\hline
\end{tabular}


Table 2 | Kellgren and Lawrence (KL) grade frequencies for pairs of knees discordant for frequent knee pain in Framingham $(n=336)$

\begin{tabular}{|c|c|c|c|c|c|}
\hline \multirow[b]{2}{*}{$\mathrm{KL}$ grades for knees without frequent knee pain } & \multicolumn{5}{|c|}{ KL grades for knees with frequent knee pain } \\
\hline & 0 & 1 & 2 & 3 & 4 \\
\hline 0 & 145 & 11 & 17 & 19 & 13 \\
\hline 1 & 11 & 12 & 8 & 8 & 1 \\
\hline 2 & 6 & 4 & 21 & 19 & 4 \\
\hline 3 & 0 & 1 & 3 & 14 & 14 \\
\hline 4 & 0 & 0 & 2 & 1 & 2 \\
\hline
\end{tabular}

4 had 1.5 (95\% confidence interval 0.9 to 2.3$), 3.9$ (2.4 to 6.5 ), 9.0 (5.2 to 15.6 ), and 151 (43 to 526 ) times higher odds of frequent knee pain, respectively, than knees with Kellgren and Lawrence grade $0(\mathrm{P}<0.001$ for trend) (fig 1). As few participants had one knee with Kellgren and Lawrence grade 4 and the contralateral knee with grade 0 , the confidence interval for this effect estimate was relatively wide. The corresponding odds ratios for Framingham participants were 1.2 (0.6 to 2.5), 3.1 (1.5 to 6.5), 15.1 (5.6 to 41.2), and 73 (16.2 to 331) $(\mathrm{P}<0.001$ for trend). Furthermore, although both osteophytes and joint space narrowing were associated with presence of frequent knee pain in a dose-response manner, the magnitude of association with joint space narrowing was stronger than that for osteophytes (fig 2).

The severity of radiographic knee osteoarthritis and individual radiographic features were also strongly associated with consistency of frequent knee pain.
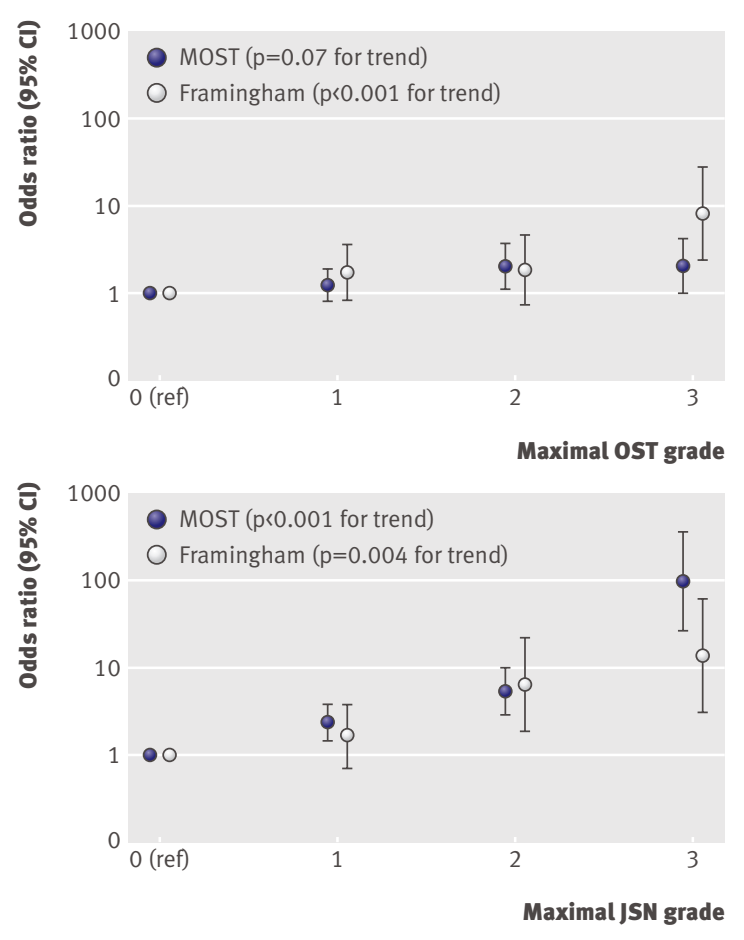

Fig 2 | Associations of maximal osteophyte (OST) and joint space narrowing (JSN) grades, mutually adjusted for one another, with frequent knee pain among people with two knees discordant for frequent knee pain status. Note that $y$ axis is logarithmically scaled
Compared with knees with a Kellgren and Lawrence grade of 0 , the odds ratios for consistent frequent knee pain versus no frequent knee pain were 1.3, 5.5, 10.0, and 317 for knees with Kellgren and Lawrence grades of $1,2,3$, and 4 , respectively $(\mathrm{P}<0.001$ for trend) (table 3 ). The odds of inconsistent frequent knee pain versus no frequent knee pain also increased as severity of radiographic osteoarthritis increased; the magnitude of association, however, was smaller than that for consistent frequent knee pain. Our findings on the relation of severity of radiographic osteoarthritis to consistent versus inconsistent frequent knee pain were similar.

As shown in table 4, severity of radiographic osteoarthritis and severity of knee pain were also positively associated. Compared with knees with Kellgren and Lawrence grade 0 , the odds ratio of severe to extreme pain versus no pain was 129 for knees with Kellgren and Lawrence grade $4(\mathrm{P}<0.001$ for trend). We found similar results for the relation of severity of radiographic knee osteoarthritis to mild to moderate pain versus no pain $(\mathrm{P}<0.001$ for trend) as well as to severe to extreme versus mild to moderate knee pain ( $\mathrm{P}<0.001$ for trend).

Furthermore, even among people in whom both knees were painful, when one knee had more severe pain than the other, as defined by differences in WOMAC pain scores (difference of $\geq 20 \%$ and absolute difference of $\geq 2$ on a 0 -20 scale), we saw similar associations. For example, increasing radiographic severity by Kellgren and Lawrence grade was associated with odds ratios of 1.0 (referent), 1.3, 2.4, 6.3, and $30.8(\mathrm{P}<0.001$ for trend) for having more severe compared with less severe knee pain. We also found similar associations for the relation of osteophytes and joint space narrowing to severity of pain, although the magnitudes of effect for osteophyte grades were not as large as those for joint space narrowing grades (data not shown).

\section{DISCUSSION}

We found a strong dose-response relation between severity of radiographic knee osteoarthritis and knee pain, as measured by three characteristics: presence of frequent knee pain, consistency of knee pain, and severity of knee pain. Moreover, we were able to show these associations even for mild stages of osteoarthritis of the knee. Our findings for the association with frequent knee pain were consistent across two large 
Table $3 \mid$ Associations of consistency of knee pain with Kellgren and Lawrence grade, maximal osteophyte grade, and maximal joint space narrowing grade among people with two knees discordant for consistency of knee pain in MOST

Odds ratio $(95 \% \mathrm{Cl})$

Consistent pain $v$ no pain Inconsistent pain $v$ no pain Consistent $v$ inconsistent $(n=429$ people $\quad(n=383$ people $\quad$ knee pain $(n=249$ people $)$

Kellgren and Lawrence grade

\begin{tabular}{lccc}
\hline 0 & 1.0 (referent) & 1.0 (referent) & 1.0 (referent) \\
\hline 1 & $1.3(0.7$ to 2.4$)$ & $1.4(0.8$ to 2.3$)$ & 2.4 (1.1 to 5.6$)$ \\
\hline 2 & $5.5(2.7$ to 11.1$)$ & $3.0(1.6$ to 5.5$)$ & $4.1(1.6$ to 10.6$)$ \\
\hline 4 & $10.0(4.8$ to 20.4$)$ & $8.6(3.7$ to 20.2$)$ & $10.2(3.7$ to 28.2$)$ \\
\hline P for trend & $317(40$ to 2523$)$ & $42.7(10.3$ to 177$)$ & $56.0(13.5$ to 232$)$ \\
\hline
\end{tabular}

Maximal osteophyte grade*

\begin{tabular}{|c|c|c|c|}
\hline 0 & 1.0 (referent) & 1.0 (referent) & 1.0 (referent) \\
\hline 1 & $0.8(0.5$ to 1.4$)$ & 1.8 (1.1 to 3.1$)$ & 1.8 (0.8 to 4.3$)$ \\
\hline 2 & $1.6(0.7$ to 3.4$)$ & 3.1 (1.4 to 6.9$)$ & 2.9 (1.0 to 8.8$)$ \\
\hline 3 & $2.0(0.8$ to 5.5$)$ & 2.3 (0.8 to 6.8 ) & 3.2 (1.0 to 10.8$)$ \\
\hline$P$ for trend & $P=0.3$ & $P=0.03$ & $P=0.1$ \\
\hline \multicolumn{4}{|c|}{ Maximal joint space narrowing grade* } \\
\hline 0 & 1.0 (referent) & 1.0 (referent) & 1.0 (referent) \\
\hline 1 & $3.0(1.7$ to 5.5$)$ & 1.7 (1.0 to 3.0$)$ & $2.2(0.9$ to 5.5$)$ \\
\hline 2 & 6.4 (2.8 to 14.2$)$ & 4.3 (1.7 to 10.7$)$ & $4.4(1.5$ to 13.0$)$ \\
\hline 3 & $103(20.4$ to 518$)$ & 26.5 (5.8 to 121$)$ & 21.1 (4.8 to 92.7$)$ \\
\hline$P$ for trend & $P<0.001$ & $P<0.001$ & $P<0.001$ \\
\hline
\end{tabular}

*Mutually adjusted for one another.

cohorts; one cohort consisted of participants with or at high risk of knee osteoarthritis, and the other was a community based sample unselected for knee osteoarthritis. Additionally, in contrast to previous findings, our study suggests that the magnitude of association between joint space narrowing and knee pain is larger than that for osteophytes and knee pain.

\section{Strengths and limitations}

Several studies have examined the association between radiographic features in the knee and knee pain across individual patients. ${ }^{4-6}$ Studies have concluded that only modest associations exist between knee pain and radiographic osteoarthritis. Although such conclusions have generally been accepted in the osteoarthritis research community, ${ }^{271113}$ they need to be scrutinised.

Firstly, confounding is a central concern in epidemiological studies. ${ }^{33}$ Unlike many other disease

Table $4 \mid$ Association of severity of knee pain with Kellgren and Lawrence grade among people with two knees discordant for knee pain severity in MOST

\begin{tabular}{lccc} 
Kellgren and & & Odds ratio $(95 \% \mathrm{Cl})$ & \\
\cline { 2 - 4 } $\begin{array}{l}\text { Lawrence grade } \\
0\end{array}$ & $\begin{array}{c}\text { Severe to extreme } \boldsymbol{v} \text { mild to } \\
\text { moderate pain }(\mathrm{n}=\mathbf{2 5 7} \text { people) }\end{array}$ & $\begin{array}{c}\text { Severe to extreme } \boldsymbol{v} \text { no } \\
\text { pain (n=64 people) }\end{array}$ & $\begin{array}{c}\text { Mild to moderate } \boldsymbol{v} \text { no pain } \\
\text { (n=533 people) }\end{array}$ \\
\hline 1 & 1.0 (referent) & 1.0 (referent) & 1.0 (referent) \\
\hline 2 & $1.5(0.6$ to 3.4$)$ & $1.2(0.2$ to 9.1$)$ & 2.1 (1.3 to 3.4) \\
\hline 3 & $2.6(1.1$ to 6.3$)$ & $7.6(1.1$ to 50.9$)$ & $6.2(3.9$ to 11.5$)$ \\
\hline 4 & $6.0(2.6$ to 14.0$)$ & $16.1(2.2$ to 115.1$)$ & $12.6(6.3$ to 25.3$)$ \\
\hline$P$ for trend & $15.7(4.8$ to 51.5$)$ & $129(8.7$ to 1908$)$ & $66.1(20.4$ to 214$)$ \\
\hline
\end{tabular}

outcomes, such as the occurrence of cancer, or pathological changes in the knee, pain is a subjective phenomenon with many factors contributing to its occurrence. Even when equal intensities of nociceptive stimuli are administered in laboratory investigations, people vary in their reports of pain and their pain related behaviour. This natural variability in responses to pain is influenced by a variety of factors and may contribute in part to variations in reports of joint pain among people with comparable pathological changes. Relatively few studies have examined the potential contribution of other such factors to the experience of pain or functional limitations in knee osteoarthritis, ${ }^{58113435}$ and a recent study contended that only a modest discrepancy exists between pain and structural pathology in osteoarthritis. ${ }^{36}$ Nevertheless, most studies do not collect sufficiently comprehensive data on all of the domains that contribute to pain to allow proper assessment of the natural variability of pain among individual people with conventional study designs. Such studies are therefore susceptible to confounding by these unmeasured and uncontrolled for factors.

In contrast to such studies, by taking advantage of nature we compared two knees within a person in whom the two knees had different levels of pain. When all person level factors influencing pain would contribute equally to both knees, we are asking why in an individual person one knee has pain (or more pain) whereas the other does not (or has less pain). This novel approach eliminates between person confounding, allowing us to obtain valid effect estimates of radiographic osteoarthritis or specific radiographic features on knee pain, even in mild osteoarthritis.

Secondly, as a substantial proportion of people with knee osteoarthritis have intermittent pain, ${ }^{37} 38$ this temporal variability further complicates observational studies of knee pain. For example, a person or knee can be misclassified as being pain-free when pain status has been ascertained at only a single time point when the person was not experiencing pain but had experienced pain recently, or vice versa. Thus, as with previous studies, our study design is also susceptible to unadjusted potential time varying factors. Nevertheless, such misclassification is likely to be non-differential in our study design and would dilute the true estimates towards the null. In MOST, we assessed participants' pain status at two proximate time points by using these two times to define consistency of knee pain. As the pathological features of osteoarthritis are generally believed not to regress, one may expect that people with more consistent knee pain would have greater underlying pathology. Indeed, in our study we found stronger associations between radiographic features of osteoarthritis in people with consistent knee pain than in those with inconsistent knee pain.

Although this study design cannot be used to assess the independent effects of person level risk factors on pain, when studying the specific structural lesions of knee in relation to pain we contend that this approach provides valid estimates of effect and insight into our 


\section{WHAT IS ALREADY KNOWN ON THIS TOPIC}

Little is understood about the causes of knee pain, as the association between radiographic osteoarthritis and knee pain has generally been accepted to be weak or modest

Previous studies comparing pain and structural abnormalities across patients may not have found a strong association because of between person confounding

\section{WHAT THIS STUDY ADDS}

Comparison of radiographic abnormalities between two knees within a person in whom pain measures in the two knees were discordant eliminated between person confounding

A strong association existed between radiographic osteoarthritis and knee pain, supporting a causative role for structural abnormalities in the presence of knee pain

understanding of the causes of pain given the complicated constellation of known and, importantly, unknown risk factors for pain.

\section{Comparison with previous studies}

In addition to the widely held belief that only a modest association exists between radiographic severity and pain symptoms, another difference compared with previous studies is that we found joint space narrowing to be more strongly associated than osteophytes with knee pain. ${ }^{112}$ This finding was consistent irrespective of which measure we used to assess knee pain. This suggests that joint space narrowing grades adequately reflect the underlying pathological changes occurring in advanced stages of osteoarthritis. Previous studies examining radiographic narrowing and its relation to pain may have been limited by radiographic techniques that either were not standardised or did not optimally and reproducibly assess joint space narrowing (such as full extension films). Given that cartilage is aneural, the ability of joint space narrowing grade to predict knee pain does not necessarily reflect solely cartilage damage; it may also reflect the concomitant change throughout the articular and extra-articular tissues. Furthermore, joint space narrowing is also a reflection of meniscal pathology, including extrusion. ${ }^{39}$ These various articular and peri-articular tissues are best imaged with magnetic resonance imaging (MRI). The study design used here affords the additional advantage of reducing the cost of MRI by limiting readings to those knees that are discordant for pain to study the association between structures visualised well on MRI and symptoms.

\section{Clinical implications}

The general belief is that if two factors do not co-occur frequently, this reflects a weak association. Many people with radiographic knee osteoarthritis may not have symptoms of knee pain. Conversely, those experiencing knee pain symptoms may not have radiographic osteoarthritis. The lack of co-occurrence of knee pain and radiographic knee osteoarthritis may suggest that radiographic osteoarthritis has limited discriminating potential for knee pain. It does not imply, however, that the association between those two factors is weak. When the aim of a study is to predict occurrence of disease, investigators are interested in building a parsimonious model that will have maximal power in discriminating people with the outcome of interest from those without such an outcome. In such circumstances, the validity of the estimate of effect of each predictor included in the model-for example, whether the effect was confounded-is not the major concern. ${ }^{40}$ To our knowledge, few, if any, epidemiological studies have attempted to overcome this problem.

What does this mean for the clinician? Just as knowing whether a patient has depressed mood does not definitively allow one to determine whether that person has pain but does contribute to the understanding of pain, knowing that a person has radiographic changes of osteoarthritis may not allow one to accurately predict the presence of pain in that person but does contribute to our understanding of pain in osteoarthritis. Understanding the pathophysiology of pain in osteoarthritis will ultimately lead to rational therapeutic targets for this disease, which has minimal treatment options.

\section{Conclusions}

In terms of tackling the conundrum of discordance between structure and symptoms, this study has confirmed that a strong structure-symptom association definitely exists in osteoarthritis of the knee. Our findings add credence to ongoing efforts to use MRI studies to better understand underlying pathological structures that may be contributing to the pain of osteoarthritis. Thus, radiographic severity, as determined by Kellgren and Lawrence grades and individual radiographic features, particularly joint space narrowing, is a strong risk factor for the presence, consistency, and severity of knee pain and accurately reflect the presence of painful pathology.

Contributors: TN, JN, and YZ were involved in conception and design of the study and in analysis and interpretation of the data. DF, MN, CEL, PA $\mathrm{BS}, \mathrm{JT}$, and LB were involved in collection and interpretation of data. TN drafted the manuscript. All authors were involved in revision and final approval of the manuscript. All authors had full access to all of the data in the study. TN and $Y Z$ are the guarantors.

Funding: MOST was funded by NIH U01 AG18820, U01 AG18832, U01 AG18947, and U01 AG19069. Framingham was funded by NIH AR47785 and AG18393 and NHLBI, Framingham Heart Study contract N01-HC25195. TN was supported by NIAMS K23 AR055127, an Arthritis Foundation arthritis investigator award, and a Boston OAIC Claude Pepper research career development core grant. The study sponsors had no role in the study design; collection, analysis, and interpretation of data; writing of the article; or the decision to submit it for publication. The researchers work independently of their funders.

Competing interests: None declared.

Ethical approval: The MOST study protocol was approved by the institutional review boards at the University of Iowa; University of Alabama, Birmingham; University of California, San Francisco; and Boston University Medical Center. The Framingham Osteoarthritis Study was approved by the Boston University Medical Center institutional review board.

1 Guccione AA, Felson DT, Anderson JJ, Anthony JM, Zhang Y, Wilson PW, et al. The effects of specific medical conditions on the functional limitations of elders in the Framingham study. Am J Public Health 1994;84:351-8.

2 Dieppe PA. Relationship between symptoms and structural change in osteoarthritis: what are the important targets for osteoarthritis therapy? J Rheum Suppl 2004;70:50-3. 
3 Hadler NM. Knee pain is the malady - not osteoarthritis. Ann Intern Med 1992;116:598-9.

4 Lawrence JS, Bremner JM, Bier F. Osteo-arthrosis: prevalence in the population and relationship between symptoms and x-ray changes. Ann Rheum Dis 1966;25:1-24.

5 Davis MA, Ettinger WH, Neuhaus IM, Barclay JD, Segal MR. Correlate of knee pain among US adults with and without radiographic knee osteoarthritis. J Rheumatol 1992;19:1943-9.

6 Hannan MT, Felson DT, Pincus T. Analysis of the discordance between radiographic changes and knee pain in osteoarthritis of the knee. J Rheumatol 2000;27:1513-7.

7 Felson DT, Naimark A, Anderson J, Kazis L, Castelli W, Meenan RF. The prevalence of knee osteoarthritis in the elderly. Arthritis Rheum 1987;30:914-8

8 Hochberg MC, Lawrence RC, Everett DF, Cornoni-Huntley J. Epidemiologic associations of pain in osteoarthritis of the knee: data from the national health and nutrition examination survey and the national health and nutrition examination-I epidemiologic follow-up survey. Semin Arthritis Rheum 1989;18:4-9.

9 Lanyon P, O'Reilly S, Jones A, Doherty M. Radiographic assessment of symptomatic knee osteoarthritis in the community: definitions and normal joint space. Ann Rheum Dis 1998;57:595-601.

10 O'Reilly SC, Muir KR, Doherty M. Screening for pain in knee osteoarthritis: which question? Ann Rheum Dis 1996;55:931-3.

11 Creamer P, Lethbridge-Cejku M, Hochberg MC. Determinants of pain severity in knee osteoarthritis: effect of demographic and psychosocial variables using 3 pain measures. J Rheumatol 1999;26:1785-92.

12 Spector TD, Hart DJ, Byrne J, Harris PA, Dacre JE, Doyle DV. Definition of osteoarthritis of the knee for epidemiological studies. Ann Rheum Dis 1993;52:790-4.

13 Lethbridge-Cejku M, Scott WW Jr, Reichle R, Ettinger WH, Zonderman A, Costa $\mathrm{P}$, et al. Association of radiographic features of osteoarthritis of the knee with knee pain: data from the Baltimore longitudinal study of aging. Arthritis Care Res 1995;8:182-8.

14 Zubieta JK, Smith YR, Bueller JA, Xu Y, Kilbourn MR, Jewett DM, et al. Regional mu opioid receptor regulation of sensory and affective dimensions of pain. Science 2001;293:311-5.

15 Mogil JS. The genetic mediation of individual differences in sensitivity to pain and its inhibition. Proc Natl Acad Sci USA 1999;96:7744-51.

16 Vase L, Riley JL 3rd, Price DD. A comparison of placebo effects in clinical analgesic trials versus studies of placebo analgesia. Pain 2002;99:443-52.

17 Colloca L, Benedetti F. How prior experience shapes placebo analgesia. Pain 2006;124:126-33.

18 Fields HL. Pain modulation: expectation, opioid analgesia and virtual pain. Prog Brain Res 2000;122:245-53.

19 Wager TD. Expectations and anxiety as mediators of placebo effects in pain. Pain 2005;115:225-6.

20 Villemure $C$, Slotnick BM, Bushnell MC. Effects of odors on pain perception: deciphering the roles of emotion and attention. Pain 2003;106:101-8.

21 Bradley LA. Recent approaches to understanding osteoarthritis pain. J Rheumatol Suppl 2004;70:54-60.

22 Giardino ND, Jensen MP, Turner JA, Ehde DM, Cardenas DD. Social environment moderates the association between catastrophizing and pain among persons with a spinal cord injury. Pain 2003;106:19-25.

23 Gamsa A. Is emotional disturbance a precipitator or a consequence of chronic pain? Pain 1990;42:183-95.
24 Deshields TL, Tait RC, Gfeller JD, Chibnall JT. Relationship between social desirability and self-report in chronic pain patients. Clin J Pain 1995;11:189-93.

25 Felson DT, Niu J, Guermazi A, Roemer F, Aliabadi P, Clancy M, et al. Correlation of the development of knee pain with enlarging bone marrow lesions on magnetic resonance imaging. Arthritis Rheum 2007;56:2986-92.

26 Englund M, Guermazi A, Gale D, Hunter DJ, Aliabadi P, Clancy M, et al. Incidental meniscal findings on knee MRI in middle-aged and elderly persons. N Engl J Med 2008;359:1108-15.

27 Felson DT, Niu J, McClennan C, Sack B, Aliabadi P, Hunter DJ, et al. Knee buckling: prevalence, risk factors, and associated limitations in function. Ann Intern Med 2007;147:534-40.

28 Karlson EW, Sanchez-Guerrero J, Wright EA, Lew RA, Daltroy LH, Katz JN, et al. A connective tissue disease screening questionnaire for population studies. Ann Epidemiol 1995;5:297-302.

29 Felson DT, Nevitt MC, Yang M, Clancy M, Niu J, Torner JC, et al. A new approach yields high rates of radiographic progression in knee osteoarthritis. J Rheumatol 2008;35:2047-54.

30 LaValley MP, McLaughlin S, Goggins J, Gale D, Nevitt MC, Felson DT. The lateral view radiograph for assessment of the tibiofemoral joint space in knee osteoarthritis: its reliability, sensitivity to change, and longitudinal validity. Arthritis Rheum 2005;52:3542-7.

31 Bellamy N, Buchanan WW, Goldsmith CH, Campbell J, Stitt LW. Validation study of WOMAC: a health status instrument for measuring clinically important patient relevant outcomes to antirheumatic drug therapy in patients with osteoarthritis of the hip or knee. / Rheumatol 1988;15:1833-40.

32 Stokes ME, Davis CS, Koch GG. Chapter 10: Conditional logistic regression. In: Categorical data analysis using the SAS system. 2nd ed. Cary: SAS Institute, 2000:271-322.

33 Rothman KJ. Epidemiology: an introduction. New York: Oxford University Press, 2002.

34 Salaffi F, Cavalieri F, Nolli M, Ferraccioli G. Analysis of disability in knee osteoarthritis: relationship with age and psychological variables but not with radiographic score. / Rheumatol 1991;18:1581-6.

35 Summers MN, Haley WE, Reveille JD, Alarcon GS. Radiographic assessment and psychologic variables as predictors of pain and functional impairment in osteoarthritis of the knee or hip. Arthritis Rheum 1988;31:204-9.

36 Duncan R, Peat G, Thomas E, Hay E, McCall I, Croft P. Symptoms and radiographic osteoarthritis: not as discordant as they are made out to be? Ann Rheum Dis 2007;66:86-91.

37 Creamer P. Current perspectives on the clinical presentation of joint pain in human OA. Novartis Found Symp 2004;260:64-74; discussion 74-8, 100-4, 277-9.

38 Hawker GA, Stewart L, French MR, Cibere J, Jordan JM, March L, et al. Understanding the pain experience in hip and knee osteoarthritisan OARSI/OMERACT initiative. Osteoarthr Cartil 2008;16:415-22.

39 Hunter DJ, Zhang YQ, Tu X, Lavalley M, Niu JB, Amin S, et al. Change in joint space width: hyaline articular cartilage loss or alteration in meniscus? Arthritis Rheum 2006;54:2488-95.

40 Janes H, Pepe MS. Adjusting for covariates in studies of diagnostic, screening, or prognostic markers: an old concept in a new setting. Am J Epidemiol 2008;168:89-97.

Accepted: 29 April 2009 